EON • Volume 15, Issue 1 - February 2022

\title{
Advocating for Yourself in the Workplace: Strategies for Taking Charge of Your Career Development
}

\section{Billy Lavelle ${ }^{1}$}

${ }^{1}$ Senior Managing Editor, J\&J Editorial

Published on: Feb 01, 2022

License: Creative Commons Attribution 4.0 International License (CC-BY 4.0). 
Have you ever tried to ride a tandem bicycle alone? You may be able to hold yourself up or even be comfortable in the seat, but attempting to travel any sort of distance on your own won't get you very far. The same could be said for advancement in the workplace. In the scholarly publishing industry, it can sometimes feel as though each of us is traveling alone. Nevertheless, the machinery of the workplace is built to function through collaboration, coordination, and communication. Honing these skills and recognizing their utility in the workplace are important first steps in advocating for yourself in the workplace and taking charge of your career development. In this article, I will discuss strategies that can aid in the ongoing pursuit of advocating for yourself, highlighting your accomplishments, communicating your goals, and working with your organization's leadership to see those goals come to fruition. Self-advocacy can take many forms, but, as I will detail, there are several general strategies you can use to help best position yourself to advance and thrive in your workplace.

The first key component of self-advocacy is building the case for yourself and your work to be considered as a prime candidate for advancement. Beginning to build your case can be tough, though, as it is often not first nature for many of us to talk ourselves up. This is where tracking and documenting your achievements in your role can come in handy. Achievements can take many forms and will likely depend on your particular role, but the strategies for tracking and documenting them are the same despite their form.

If you are early in your career or otherwise at a loss as to what achievements are worth documenting, some of the most tangible and consistent evidence of excellence you can demonstrate is positive feedback from the teammates, clients, or other stakeholders you interact with in your role. Who could better detail the fantastic work you do than the people who see and benefit from it every day? Receiving positive feedback can be validating, and even make your day, but it can also be easy to file away after reading and move on to the next item on your to-do list, assured that you've done a good job. Positive feedback can offer much more than a temporary ego boost, however. It can be indispensable in tangibly demonstrating your utility within your organization.

The ways in which one can track and document your achievements are numerous and dependent on your work style. One strategy is to create a designated folder on your 
desktop for storing positive feedback or other documented achievements so that it can easily be accessed at the appropriate time. Similarly, if your role requires extensive use of email, you can create a sub-folder in your inbox for saving emails with positive feedback or interactions you feel show your work in a positive light. A third approach is to keep a running "resume" of accomplishments and feedback that can be used for reference or shared with contacts within your organization when needed. All of these approaches have their utilities and can be beneficial in different ways. What is important is that you find an organizational method that works best for you. Storing your accomplishments and positive feedback to be organized and accessible at a moment's notice will allow you to be prepared when opportunities for new roles or advancements arrive.

However, keeping track of your achievements is only the first step in the journey. One can have all the positive feedback in the world, but its utility is relative to one's ability to strategically utilize it. This is where the importance of seeking out and leveraging opportunity comes in. A major element of leveraging opportunity is to first develop an understanding of the structure and "chain of command" within your organization. Ask yourself: Who assigns new projects in your organization? Do you interact with that person or those people regularly? If not, are there opportunities for you to meet with them more often? Finding positive answers to these questions can help direct your efforts so that they are not in vain.

Once you have answered these questions, a new, more daunting one may arise: How do I make a persuasive case for myself when meeting with leadership? While the answer to this question may depend on the structure of your organization and who you are meeting with, there are a few general tips you can keep in mind. Most paramount is to plan ahead and be prepared to talk about what is most important to you. First, consider the goals you have for your career and how best to communicate them. Next, consider both what you can do to attain those goals and what support you need from your organization to achieve them. It may be worth writing down your thoughts in advance of any meeting so that you do not lose track once the conversation begins. Once you are prepared, it is also important to be honest and open to feedback. Be forthright about any challenges you are encountering, but also be receptive to any concerns or areas for improvement. Addressing these can be among the surest ways to position yourself for advancement. By being prepared, being forthright, and being open to feedback, you will be able to make the most of your meetings with leadership and leverage opportunities that come your way. 
Related to leveraging opportunity is the importance of getting your name out and putting yourself on the respective radars of those in your organization responsible for identifying and fostering talent. Often in the scholarly publishing industry, we work in silos where intersection with people in other roles, other organizations, or even our direct colleagues is not always frequent. An essential aspect of self-advocacy in our industry is recognizing the ways in which siloing occurs and compensating for this through deliberate interaction. By taking advantage of opportunities within your organization to meet and interact with others outside of your immediate orbit, you will multiply your chances and outlets for growth.

While it can be easy to feel isolated from others not associated with our day-to-day duties, most organizations have opportunities for people to connect with one another outside of their regular workday tasks. These can take the form of internal initiatives, such as committees or planning of internal events, opportunities to volunteer for new or additional work, or even membership in external organizations like ISMTE. Alternately, even during COVID, many workplaces create opportunities for employees to interact socially either during or outside of operating hours. While these opportunities are typically not required, taking advantage of them and establishing yourself as a reliable presence within your organization's culture will open doors that may otherwise have remained locked. In addition, presenting yourself as a dependable resource not only to those you work with on a day-to-day basis but also to others within and outside your organization will help ensure your name is top-of-mind for as many people within your organization as possible when new opportunities arise.

Undertaking the above strategies can be challenging enough in a traditional office environment, but the increasing universality of remote work creates new challenges that can complicate our ability to advocate for ourselves in the same ways we did before the pandemic. While the transition to remote work has been disruptive for many, the changes in how we communicate during the workday present new and distinct opportunities for self-advocacy in the workplace. Perhaps the most pronounced difference between the world of remote work and the office is the necessity of being intentional with our communication. Unlike in the office, where opportunities could arise from chance encounters and casual conversations, the onus is now upon each of us to communicate with intention. If we want to connect with someone, we must take specific action to do so; we can no longer rely on the water cooler or incidental meetings around the office. 
While this may be more comfortable for some, it also presents a unique challenge for self-advocacy. It theoretically reduces the number of opportunities we would otherwise have to share our achievements, leverage opportunity, and get our names out. The transition to remote work does not necessarily change any of these strategies, but it does mean that we must be more thoughtful and deliberate when pursuing them. Because leadership is less able to see the outcomes of your work remotely, it becomes even more critical to take ownership of tracking and documenting your achievements. Because opportunities may come at a more infrequent pace remotely, it is more essential to leverage those that remain in the most fruitful way possible. And, because it is harder to get your name in out in a virtual environment, it becomes more crucial to connect and network with those you don't interact with on a regular basis. If anything, the move to remote work only underlines the need for strategies such as these to help navigate the evolution of the workplace.

While not exhaustive, the strategies I have detailed in this article are effective first steps in the journey of taking charge of your career development. By documenting your achievements, leveraging opportunity, getting your name out, and understanding the ways in which remote work has transformed the workplace, you will place yourself in an ideal position to attain your career goals. For those who have already taken these steps or are otherwise curious, some additional, related tips are:

- Be proactive: The brave new world of remote work is one in which opportunities may not arrive by chance in the way they did in person. It is essential to think ahead and strategize for the ways in which you can make the case for yourself within your organization.

- Diversify your skillset: Keep in mind that the more diverse your skillset is, the more opportunities you will be able to seize. Consider ways in which you can learn new skills or creatively apply your current skills to new scenarios. Also consider the needs of your organization and think about ways in which you can use your current skills or develop new ones to help meet those needs.

- Be assured that your path is your own: As difficult as it can be, it truly is best to avoid making direct comparisons between your own career trajectory and those of others. Remember that opportunities come fast and often to those who seek them out.

With these tools in mind, the journey to reach the preferred outcome of your career may be the same, but you will not need to ride the tandem bicycle alone. 


\section{Disclosure Statement}

The author has no conflicts of interest to disclose. 